\title{
A Measurement System for Ultrawide-Band Communication Channel Characterization
}

\author{
Kamal Sarabandi, Fellow, IEEE, Nader Behdad, Student Member, IEEE, Adib Nashashibi, Senior Member, IEEE, \\ Mark Casciato, Member, IEEE, Leland Pierce, Senior Member, IEEE, and Feinian Wang, Student Member, IEEE
}

\begin{abstract}
In this paper a novel wide-band propagation channel measurement system with high dynamic range and sensitivity is introduced. The system enables the user to characterize signal propagation through a medium over a very wide frequency band with fine spectral resolution (as low as $3 \mathrm{~Hz}$ ) by measuring the attenuation and phase characteristics of the medium. This system also allows for the study of temporal, spectral and spatial decorrelation. The high fidelity data gathered with this system can also be utilized to develop empirical models or used as a validation tool for physics based propagation models which simulate the behavior of radio waves in different environments such as forests, urban areas or indoor environments. The mobility and flexibility of the system allows for site specific measurements in various propagation scenarios.
\end{abstract}

Index Terms-Attenuation measurement, communication channels, electromagnetic measurements, microwave measurements, radio propagation.

\section{INTRODUCTION}

$\mathbf{I}$ $\mathrm{N}$ order to assess the performance of any communication system it is necessary to characterize the communication channel. Channel characterization can be done in two ways: experimentally and by computer simulation. Some of the more commonly used attenuation models are heuristic, based on measured data [1], [2], and in some cases overly simplified electromagnetic formulations [3], with little correlation to the physics of the problem. Thus, while simple to implement, these models lack the desired accuracy and generality.

In recent years significant efforts have been devoted towards the development of physics-based electromagnetic (EM) models, which apply analytic and numeric techniques for channel modeling [4]-[13]. These models allow for characterization of the communications channel to a high degree of fidelity, and are able to simulate varied propagation environments, including the polarimetric aspects of the channel.

Despite significant progress in this area, experimental characterization of a communications channel is still necessary for gaining insight into the mechanisms of wave propagation as well as for the validation of computer models. Past experimental work on channel characterization has been carried out

Manuscript received September 27, 2003; revised August 26, 2004. This work was supported by the DARPA FCS Communication Project under subcontract S01-19 from Pennsylvania State University.

K. Sarabandi, N. Behdad, A. Nashashibi, L. Pierce, and F. Wang are with the Radiation Laboratory, Department of Electrical Engineering and Computer Science, The University of Michigan, Ann Arbor, MI 48109-2122 USA (e-mail: saraband@umich.edu).

M. Casciato is with EMAG Technologies, Incorporated, Ann Arbor, MI 48105 USA.

Digital Object Identifier 10.1109/TAP.2005.850705 often over limited bandwidths [14]-[19], and at times for specific systems such as AMPS and PCS wireless communication systems [20]. In these experimental efforts, limited channel characteristics such as path loss and fading statistics have been determined. Although channel characterization over a narrow bandwidth is straight forward, measurements over a wider bandwidth, and coherently, is significantly more complex. Basically, the problem stems from the fact that coherent receivers with a wide bandwidth allow significant noise power to enter the receiver, which limits the system's dynamic range. Measurement systems employing a single vector network analyzer (VNA) have been proposed for indoor channel characterization efforts [21]-[24]. With this approach, coherent, wideband, propagation measurements can be performed over relatively short distances. To maintain coherence, the signal generated by the network analyzer is relayed to the distant transmitter via long RF cables or optical links [24]. While this approach performs well in an indoor setting, it is impractical for an outdoor setting where multiple sites need to be accessed, often behind significant foliage. To circumvent this problem a novel approach, based on a stepped frequency technique is developed. The system uses two VNAs, one as a transmitter and one as a receiver and is synchronized in frequency step by using two rubidium atomic clocks. The sensitivity of the receiver is determined by the intermediate frequency (IF) filter internal to the VNA which can be set a low as $3 \mathrm{~Hz}$. The combination of the narrowband IF filter and the synchronized frequency sweep allows for a highly sensitive measurement system, and operation over a very wide bandwidth, in principle limited by the antennas and the specific VNAs used. In Section II, the design and operating principles of the system will be discussed, while in Section III results from a measurement campaign, conducted at the Lakehurst Naval Air Station, in Lakehurst, $\mathrm{NJ}$, are presented and analyzed, including techniques used for post processing the data and extracting the attenuation characteristics of the channel, as well as its frequency decorrelation.

\section{WIDE-BAND MEASUREMENT SYSTEM}

A VNA is a microwave measurement instrument capable of measuring both the amplitude and phase of the reflection and/or transmission coefficients of the device under test (DUT). In addition to different processing and calibration units, any VNA has a microwave signal generator, which can be either in the form of an analog sweeper or a synthesized source, and a number of built in, high performance vector receivers capable of measuring both the amplitude and phase of the input signal. The microwave sweeper allows for rapid acquisition of the signal in 


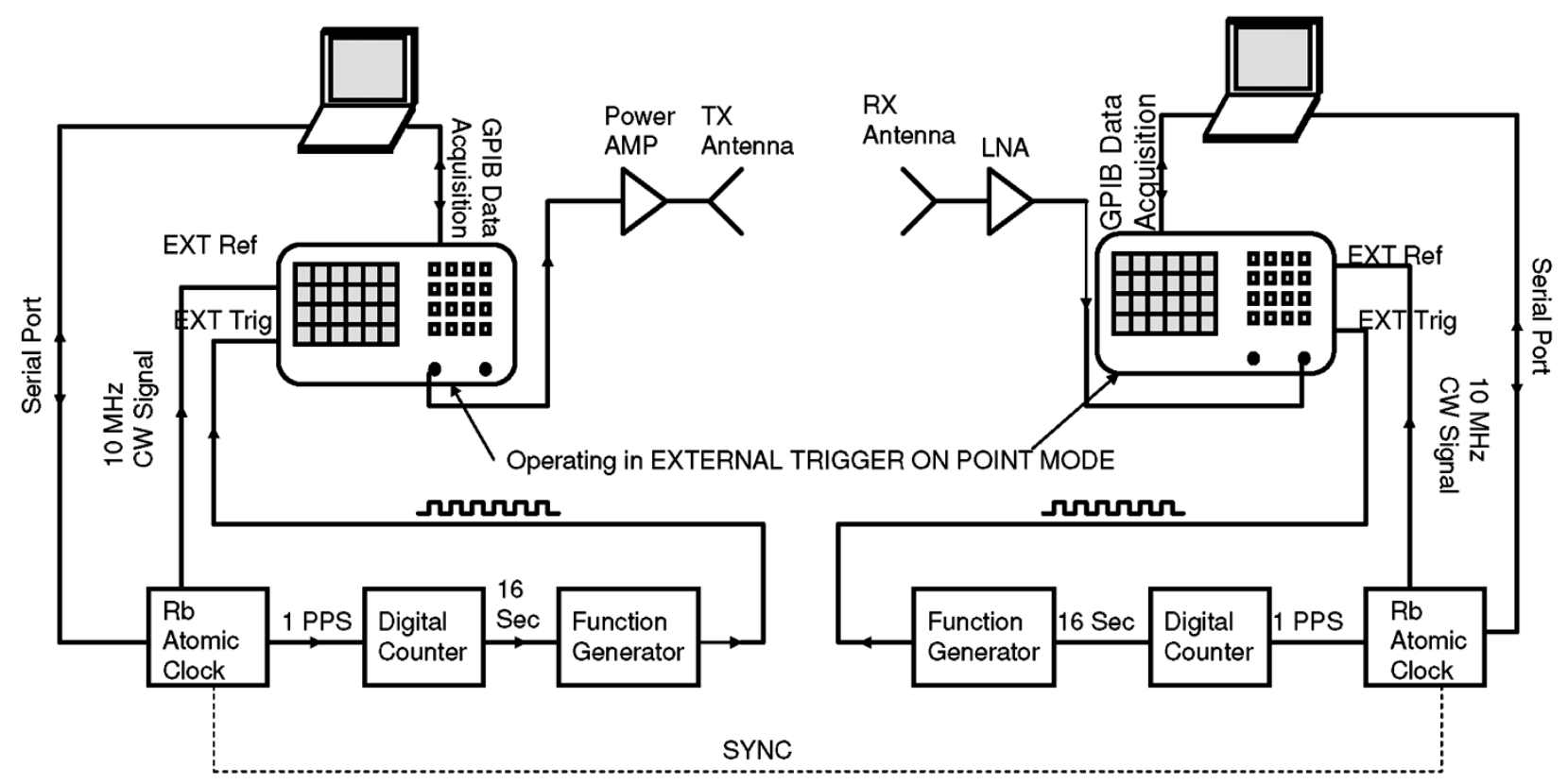

Fig. 1. Block diagram of the propagation measurement system.

broadband measurements, and also provides a constant output power over the entire frequency band. For accurate phase measurements, the receiver uses a phase lock loop (PLL) and either an internal or an external source as a reference signal for the PLL. Most VNAs such as the HP8753D or HP8720D have the option to use an external continuous wave (CW) reference (usually a $10 \mathrm{MHz}$ sinusoidal wave). This allows the user to select a highly stable $\mathrm{CW}$ signal as a frequency reference, in order to achieve high accuracy.

A communication channel can be viewed as a two port passive device with input and output ports located at the transmitter and receiver, respectively. The advantage of using a VNA as the transceiver is its stepped frequency mode of operation, and its inherent ability to sweep over a wide frequency band (50 MHz-20 GHz for an HP8720D) while maintaining a high receiver sensitivity. To maximize receiver sensitivity the receiver bandwidth (IF filter) can be set as low as $3 \mathrm{~Hz}$ while sweeping through a wide frequency band. This allows for coherent measurements (magnitude and phase) of very weak signals over a wide dynamic range. Unlike most two port devices however, the ports of this system are distant from each other and in order to perform transmission $\left(S_{21}\right)$ measurements it is necessary to employ two VNAs, one as a transmitter (Tx) and one as a receiver ( $\mathrm{Rx})$. In addition, amplifiers, antennas, filters and data acquisition systems are also needed. The difficulty of this type of disconnected system is in providing a common, stable reference to the PLLs of both VNAs, and also in synchronizing their respective frequency sweeps. Both of these difficulties are solved by using two identical, highly-stable, and synchronized Rubidium Atomic Clocks (RbAC) [25]. Synchronization of the time references of these atomic clocks is accomplished by simply connecting the time reference output of one to the time reference input of another, and once synchronized they each provide what is essentially a common, and highly stable 1 pulse per second (pps) system clock (which synchronizes the respective frequency sweeps). In addition, the RbACs also provide an ultralow noise $10 \mathrm{MHz}$ highly accurate and stable frequency standard which disciplines an ovenized oscillator to a hyperfine transition in the ground state of rubidium. This $10 \mathrm{MHz} \mathrm{CW}$ reference is used as the reference for the VNA PLLs. The short term and long term stability of these atomic clocks are, respectively, $1 \times 10^{-12} / 10 \mathrm{sec}$ and $5 \times 10^{-10} /$ year.

In order to measure the channel response correctly, both the transmitter and the receiver must synchronously sweep through the frequency band, with the allowed timing error within the $\pm 3 \mathrm{~dB}$ bandwidth of the receiver IF filter, around the frequency being measured. For large propagation delays, which cause a timing error outside this allowed range, a delay can be introduced in the timing of the receiver RbAC, to account for this. This delay is controlled by the serial port line to the data acquisition computer, shown in the system block diagram in Fig. 1. In results shown later however, distances of up to $1.1 \mathrm{~km}$ were measured without a need for any timing adjustments to the receiver clock. The atomic clocks could be used to trigger each sweep through the frequency band and the VNAs allowed to then independently sweep through the band. There is however, sufficient difference in the nominal sweep times of each independent VNA, even when set to be identical, to cause the system to loose synchronization. Therefore the atomic clocks are used to trigger a function generator, which sends a burst of TTL compatible pulses to the VNA and with each pulse moves the VNA, step by step, through each discrete frequency point to be measured in the band. This is accomplished by operating the VNAs in the "External Trigger On Point" mode. In this mode an external trigger is required, at the "EXT TRIG" input of the VNAs, to step the network analyzers through each discrete frequency point in the measurement band. This can be seen in Fig. 1, which shows a block diagram of the measurement system. To implement this for both the Tx and Rx VNAs, and referring to Fig. 1, the 1 pps 




Fig. 2. Flow chart of the system operation.

output of the rubidium atomic clock is used as a system clock, which is then fed to a digital counter that creates a time reference (in this case, by dividing it by 16) which is used to set the period of the frequency sweep (16 s). The $1 \mathrm{pps}$ time reference from $\mathrm{RbAC}$ in turn signals a function generator to send a burst of $N$ trigger pulses to the "EXT TRIG" port of the VNA with the rate of $\nu$ pulse per second (where $N$ is the total number of frequency points). Thus, a frequency sweep is performed in $t=N / \nu$ seconds. The sweep can be performed over a continuous frequency band, or the VNAs can be programmed to operate at discrete sub-bands, with non-uniform frequency sampling. This is particularly useful for conducting measurements in places where transmission over certain frequency bands or at certain frequencies with in the measurement band are not allowed, for example near or within airports, military bases, etc.

Fig. 2 shows the flowchart of the system operation. To summarize system set-up and operation, and referring to Fig. 2, the first step is to synchronize the $1 \mathrm{pps}$ outputs of the transmitter and receivers of the $\mathrm{RbACs}$ so that the $\mathrm{Tx} / \mathrm{Rx}$ time references are the same. This can be done simply by feeding the 1 pps output of one of the clocks to the synchronization input of the other one [25]. Every $16 \mathrm{~s}$ the function generator receives a trigger signal from the digital counter and sends a burst of $N$ pulses (TTL compatible) to the VNA with the rate of $\nu$ pps. These are the trigger signals, denoted "trigger" in Fig. 2, which causes the transmitter VNA to start stepping through the desired frequency band. The same thing happens simultaneously at the receiver end, except that the receiver VNA begins to receive the signal that has been transmitted by the Tx VNA. After each frequency sweep the $\mathrm{Tx} / \mathrm{Rx}$ computers acquire the measured data for post processing. In order to ensure that a new sweep does not begin while this data acquisition (DAQ in Fig. 2) is in progress, both VNAs are operated in single sweep, as opposed to continuous sweep, modes. This mode requires that each VNA be armed by the DAQ computer, before they will accept any trigger signal for a frequency sweep, thereby allowing the DAQ computer to hold the measurement cycle, while DAQ is in progress.

\section{A. Calibration, and Operation}

The received signal power can be obtained using the modified Friis transmission formula

$$
P_{R}=\frac{P_{T} G_{T} G_{R}}{L_{\mathrm{FS}} L_{A}}
$$

where $P_{T}, G_{T}$, and $G_{R}$, are the transmitted power, and the antenna gains of the transmitter and receiver, respectively, $L_{\mathrm{FS}}$ is the free space loss and $L_{A}$ is the additional path loss in the channel. The noise power at the input of the receiver VNA is calculated using:

$$
N_{R}=K T B_{\mathrm{IF}}
$$

where $K$ is Boltzman's constant, $B_{\mathrm{IF}}$ is the IF bandwidth of the VNA that can be set by the user. For the case of HP8753D this bandwidth can vary from 3 to $3.7 \mathrm{kHz}$. In (2), $T$ is the equivalent noise temperature of the system including the antenna noise temperature. A desired received signal-to-noise ratio, defined as $\mathrm{SNR}_{R}=P_{R} / N_{R}$, determines system compatibility in the path loss measurements. For most practical purposes a $\mathrm{SNR}_{R}$ of $15 \mathrm{~dB}$ is sufficient for accurately determining the statistical behavior of the channel. Referring to (2), noise power is effected by both the system bandwidth, set by the IF bandwidth of the receiver, and by the equivalent noise temperature, $T$, which is usually determined by the noise figure (NF), of the first receiver amplifier, or pre-amplifier. Therefore, the system $\mathrm{SNR}_{R}$ can be significantly reduced by reducing the IF bandwidth of the receiver VNA, but as a result of this the sweep time and the overall measurement time is increased. Hence, depending on the output power, distance, expected path loss etc., there is tradeoff between the IF bandwidth, sweep time, which determines the speed at which measurements can be taken, and the desired $\mathrm{SNR}_{R}$. Taking this into account, the IF bandwidth for the system was set to $3 \mathrm{kHz}$. This in combination with a low-noise pre-amplifier (LNA), with a NF of $1.5 \mathrm{~dB}$ and a gain of $27 \mathrm{~dB}$, resulted in a noise floor for the receiver of $-90 \mathrm{dBm}$. In addition, due to the long distances over which this measurement system will be applied, high power radio frequency (RF) amplifiers were used to amplify the transmitted signal.

To perform measurements over a wide frequency band, it is necessary to use either a very wide-band antenna, or to use different antennas for measuring different sub-bands. The gain and radiation characteristics of the antenna(s) used in the system as well as the frequency responses of the power amplifier, LNA and cables used, should be measured over the entire frequency band so that their effect can be calibrated out. Two different methods for calibration of the system are used. In the first method all of the system components' (cables, amplifiers, etc.), gains and losses are characterized individually using the VNA and then effects are accounted for in the calibration process. In the second method, which is illustrated in Fig. 3, the output of the power amplifier is connected to the input of the LNA via a high power calibrated attenuator and the overall 


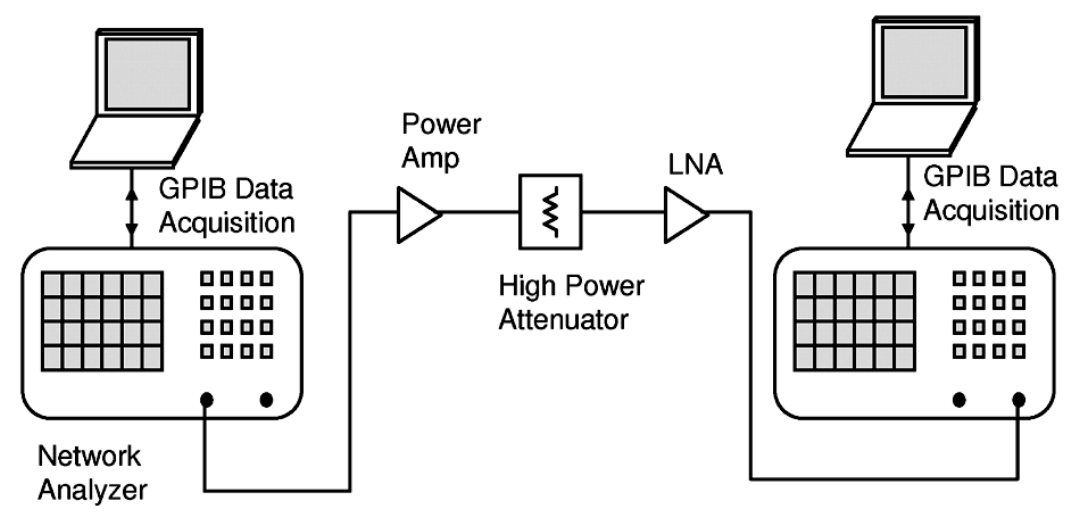

Fig. 3. Block diagram of the calibration set-up of the system.

TABLE I

Allowed Transmission Frequencies at the Lakehurst Base in Terms of Center Frequency $f_{0}$, And Bandwidth, BW. Frequencies ARE TransmitTEd at Discrete $1 \mathrm{MHz}$ INTERVALS ACross EACH RANGE SPECIFIED

\begin{tabular}{c|c|c|c|c|c|}
\hline \multicolumn{2}{c|}{ Band 1: $30 \mathrm{MHz}$ to $200 \mathrm{MHz}$} & \multicolumn{2}{c|}{ Band 2: $200 \mathrm{MHz}$ to 1 GHz } & \multicolumn{2}{c|}{ Band 3: $1 \mathrm{GHz}$ to 3 GHz } \\
\hline \hline$f_{0}(\mathrm{MHz})$ & BW $(\mathrm{MHz})$ & $f_{0}(\mathrm{MHz})$ & BW $(\mathrm{MHz})$ & $f_{0}(\mathrm{MHz})$ & BW (MHz) \\
\hline 38 & 16 & 325 & 10 & 1747 & 32 \\
\hline 70 & 16 & 350 & 32 & 1872 & 32 \\
\hline- & - & 390 & 32 & 2440 & 46 \\
\hline- & - & 435 & 25 & 2550 & 10 \\
\hline- & - & 493 & 46 & - & - \\
\hline- & - & 524 & 8 & - & - \\
\hline- & - & 561 & 8 & - & - \\
\hline- & - & 600 & 8 & - & - \\
\hline- & - & 641 & 8 & - & - \\
\hline- & - & 670 & 48 & - & - \\
\hline- & - & 806 & 8 & - & - \\
\hline- & - & 915 & 46 & - & - \\
\hline
\end{tabular}

transmission coefficient $\left(S_{21}\right)$, without the interlying propagation medium, is determined. In this approach all components except the antennas, are characterized and the method has the advantage of calibrating out the effects of connector losses and mismatches. The antennas used in this system are calibrated standard gain antennas with linear polarization and relatively low gains (wide beam). As is true for most wide-band antennas the location of the phase center of the antenna changes with frequency but the change is less than the extent of the antenna structure. This dispersive behavior of the antenna can cause spectral decorrelation to some extent but it is much lower than what is caused by the channel, and can therefore be ignored.

\section{MEASUREMENT CAMPAIGN AT LAKEHURST}

In this section a measurements campaign, using the coherent propagation measurement system and conducted at the Lakehurst Navel Air Station, Lakehurst, NJ, will be described. Results will be shown, including system performance specifications, mean path loss (PL) in terms of power, and frequency coherence analysis.

\section{A. Measurement Parameters}

The measurements at Lakehurst were performed in the $30 \mathrm{MHz}$ to $3 \mathrm{GHz}$ range in three sub-bands, 30 to $200 \mathrm{MHz}$ (defined as Band 1), $200 \mathrm{MHz}$ to $1 \mathrm{GHz}$ (defined as Band 2), and 1 to $3 \mathrm{GHz}$ (defined as Band 3). These restricted spectrums were limited by the authorities at the Lakehurst base and shows the system's capability to operate in arbitrary sub-bands within the system bandwidth. A $10 \mathrm{~W}$ power amplifier, Amplifier Research (AR) model 10W1000, was used for the transmission of frequencies at and below $1 \mathrm{GHz}$, and a $30 \mathrm{~W}$ amplifier, AR model $30 \mathrm{~S} 1 \mathrm{G} 3$, was used for transmission in the 1 to $3 \mathrm{GHz}$ band. For efficient transmission, different antennas were used in each sub-band. EMCO bowtie antennas, models 3109 (rated at $2 \mathrm{~kW}$ continuous wave $(\mathrm{CW}$ ) power) and $93110 \mathrm{~B}$ (from $\mathrm{HP}$ ) were used for the transmit and receive antennas in Band 1, respectively. EMCO models 3148 and 93146 (also from HP) log-periodic antennas were used for Band 2, and two EMCO model 3115 horn antennas for Band 3. Due to transmission restrictions at Lakehurst, the transmissions frequencies were limited to specific frequencies and bandwidths within each sub-band. Table I lists the allowed transmission frequencies 


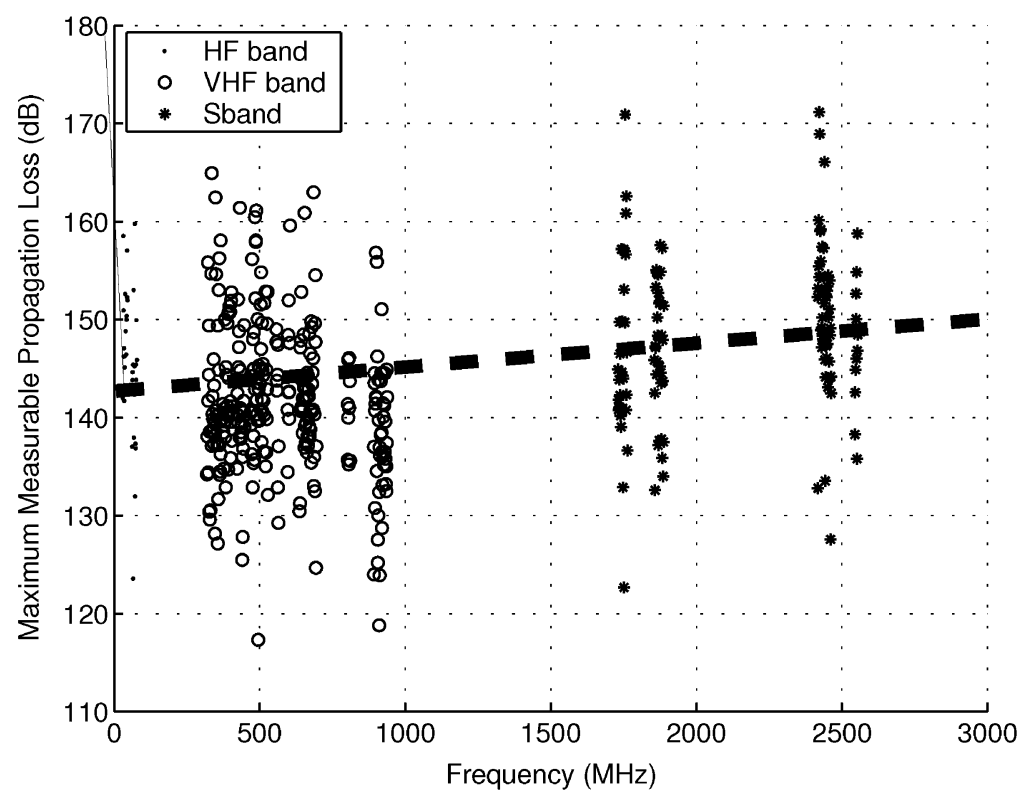

Fig. 4. Measurable propagation loss of the system.

in terms of center frequency, $f_{0}$, and bandwidth, $\mathrm{BW}$, for each allowed transmission band (Note that within the ranges specified in Table I transmission frequencies were at discrete $1 \mathrm{MHz}$ intervals).

\section{B. Measurement Results}

Before beginning an analysis of the measured data taken at the Lakehurst site, it is important to note the capabilities of the coherent measurement system. Fig. 4 shows an example of the dynamic range of the measurable propagation loss between the transmitter and receiver for the system, through the entire range that measurements were taken. This is essentially a measure of system sensitivity, and can be improved by further narrowing the IF bandwidth of the receiver. To determine this maximum measurable propagation loss, the receiver signal is sampled, with no transmitter signal present, and normalized to the transmitter power, i.e., Fig. 4 shows the receiver noise power (including the LNA pre-amplifier), referenced to the transmitter power when the IF bandwidth of the receiver VNA is set at $3 \mathrm{kHz}$. Fig. 4 is normalized, assuming a transmitter power of $10 \mathrm{~W}$. In Fig. 4, data was taken at each frequency point in the measurement band. The change in power levels observed across the band are typical of noise power fluctuations in a measurement system. The dashed line in the figure is a best fit curve, which is provided as a reference. As can be seen in this figure, the dynamic range of the measurable propagation loss across the measurement band is in excess of $115 \mathrm{~dB}$, for the IF band setting of $3 \mathrm{kHz}$.

For all measured results shown, the transmitter was at a fixed location, with data from two receive sites provided here. At each receiver location, approximately 100 spatial samples were taken on a line perpendicular to the line between transmitter and receiver. For each sub-band the spatial samples were taken over a range of $50 \lambda$ at the lowest frequency in the sub-band, in order to capture the effects of slow fading. To capture the effects of fast fading, the sampling increments began at $1 / 2 \lambda$ increments

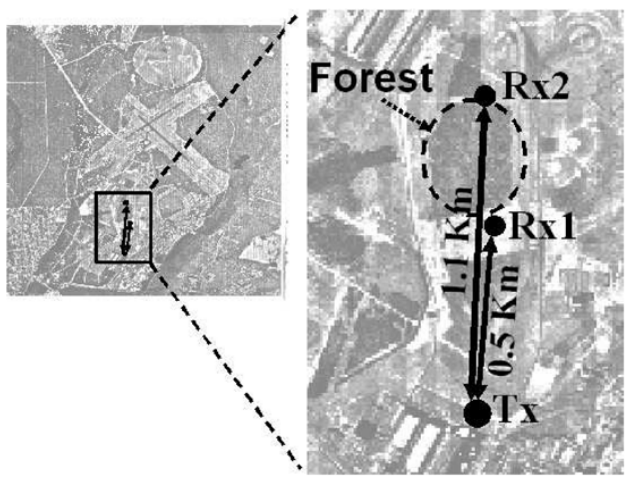

Fig. 5. Lakehurst measurement site.

at the highest frequency in the sub-band, gradually increasing to $1 / 2 \lambda$ at the lowest frequency sub-band.

The forested areas at the Lakehurst site consisted mainly of pitch pine, with some short vegetation in between. The pine trees had an average tree density of $0.113 \mathrm{Trees} / \mathrm{m}^{2}$, average height of $9 \mathrm{~m}$, average crown depth of $5 \mathrm{~m}$, and an average trunk diameter of $16 \mathrm{~cm}$.

The left side of Fig. 5. shows an aerial view of the Lakehurst site. On the right side of Fig. 5, the transmitter location, along with the two receive sites, is pictured. The transmitter site is located at the bottom of the photo, and is marked as Tx. The receive sites, designated $\mathrm{Rx} 1$ and $\mathrm{Rx} 2$ in Fig. 5, are located vertically from the Tx site, at distances of approximately 490, and $1120 \mathrm{~m}$, respectively. Note that the area between the Tx and Rx 1 is clear of forest, while the area between $\mathrm{Rx} 1$ and $\mathrm{Rx} 2$ consists of approximately $100 \mathrm{~m}$ of clear area, and $530 \mathrm{~m}$ of forest, with Rx1 located at approximately $100 \mathrm{~m}$ from the forest, and Rx 2 located on the back side of the forest, within $2 \mathrm{~m}$ of the forest. The transmitter and receiver at all locations, and for all bands measured is set at $2 \mathrm{~m}$ height. Unmarked areas are clear of forest, and may include some short vegetation. 


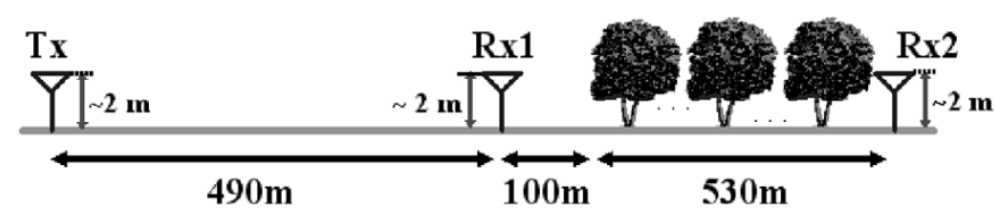

(a)

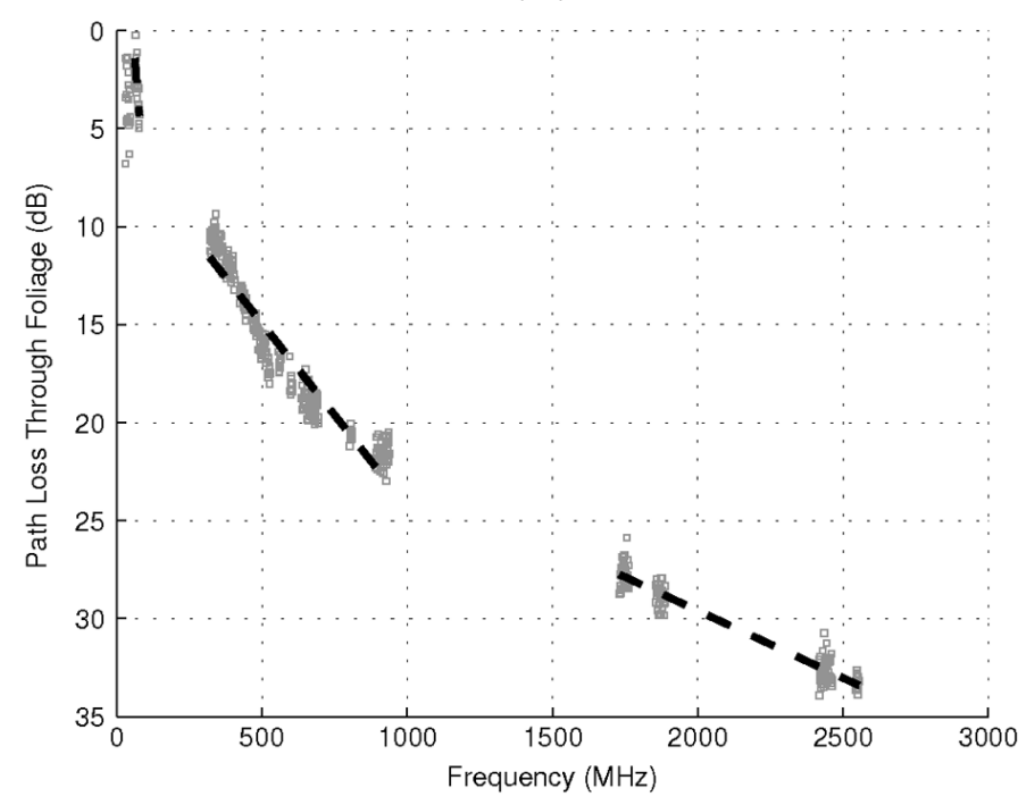

(b)

Fig. 6. Path-loss through forest: (a) measurement scenario; (b) path loss above free space, Rx2 referenced to Rx1.

In order to isolate the effects of the forest on PL, the measured data taken at Rx2, is normalized to that taken at Rx1. Fig. 6(a) shows the measurement scenario, with Fig. 6(b) showing the $\mathrm{PL}$ at $\mathrm{Rx} 2$, referenced to that at $\mathrm{Rx} 1$. The received power $P_{r}$, referenced to the transmitter power $P_{t}$, is given by

$$
\frac{P_{r}}{P_{t}}=\frac{\left(G_{t} G_{r}\right)^{2}}{\left(4 R^{2}\right)} \frac{1}{R^{m}} 1 / \mathrm{PL}
$$

where $G_{t} G_{r}$ are the transmit and receive antenna gains, respectively, $R$ is the distance between transmitter and receiver, PL is the PL of the forest, and $m$ is an attenuation factor caused by the ground effect. Note that the first factor in (3) corresponds to the free space PL, as previously given. This ground effect can vary from 0 for a very rough ground, to 2 , for cases of transmit and receive antennas very close to a perfectly flat earth. If $R_{1}$ and $R_{2}$ are the distances to $\mathrm{Rx} 1$ and $\mathrm{Rx} 2$ respectively, the power received at $\mathrm{Rx} 2$ referenced to that received at $\mathrm{Rx} 1$ is given by

$$
\frac{P_{r 2}}{P_{r 1}}=\left(\frac{R_{1}}{R_{2}}\right)^{m+2} 1 / \mathrm{PL}_{\text {forest }}=\left(\frac{490}{1120}\right)^{m+2} 1 / \mathrm{PL}_{\text {forest }}
$$

where $\mathrm{PL}_{\text {forest }}$ is the effects of the foliage on PL.

Referring to (4), Fig. 6(b) shows the PL of Rx2, referenced to that of Rx1, or the path loss above free space, for the area in between, which consists mostly of the forested area. As can be seen in Fig. 6(b), as frequency is increased, the path loss increase rate decreases. This bending effect, is due to the contribution of higher order scattering on the mean power. At low frequencies, the dominate contributor to the mean power is from single scattering (forward scattering, represented by Foldy's theory, [26]). As frequency is increased, multiple scattering among leaves and branches, which decays at a lower rate than single scattering, begins to dominate the mean power, thus the bending observed in Fig. 6(b).

\section{Frequency Correlation Analysis}

In the wide-band measurement system described in the previous sections, the frequencies of the phased lock loops (PLLs) of the two network analyzers are locked together but their phases are not. What this means, is that at the start of each frequency sweep, the absolute phases of the two locked local oscillators are unknown. As will be shown the difference in the absolute phase between the Tx and Rx VNAs is in the form of a linear phase shift, for each spatial sample, across the measured frequency band. This is similar to the phase shift that is caused by the propagation of the signal through free space. The $10 \mathrm{MHz}$ reference signal from the RbAC signal generator can be expressed as $\cos \left(\omega_{10} t+\phi_{r}\right)$. Where, $\omega_{10}=2 \pi \times 10^{7}$ and $\phi_{r}$ is the random phase of the oscillator. The VNA's synthesizer uses this reference to generate the desired frequencies in the sweep operation. The instantaneous frequency can be expressed as

$$
\omega_{\text {ins }}=\alpha(t) \omega_{10}+\alpha^{\prime}(t) \phi_{r}
$$

and the instantaneous phase is $\alpha(t) \phi_{r}$. Since a linear frequency sweep as a function of time is being performed, $\alpha(t)$ is a linear 


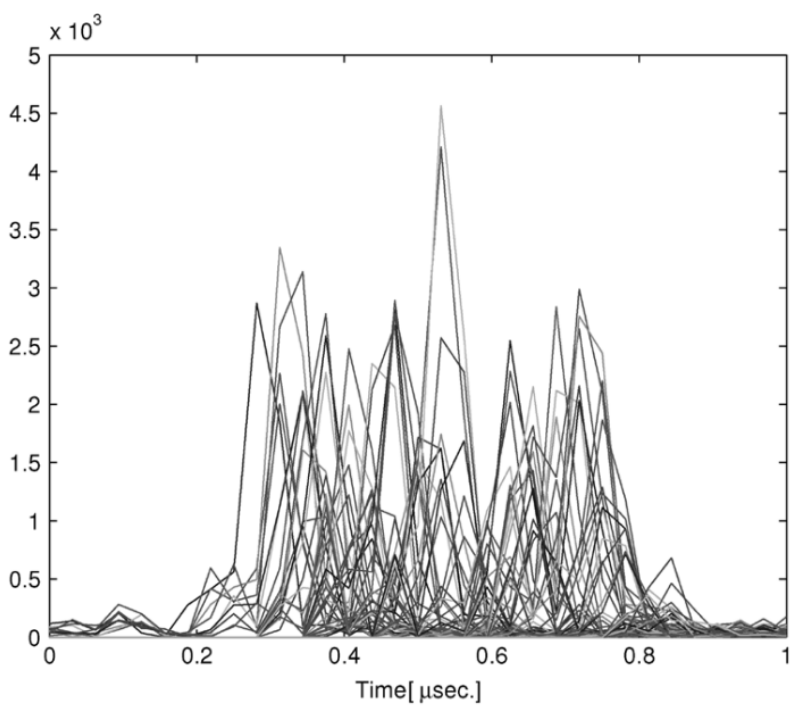

(a)

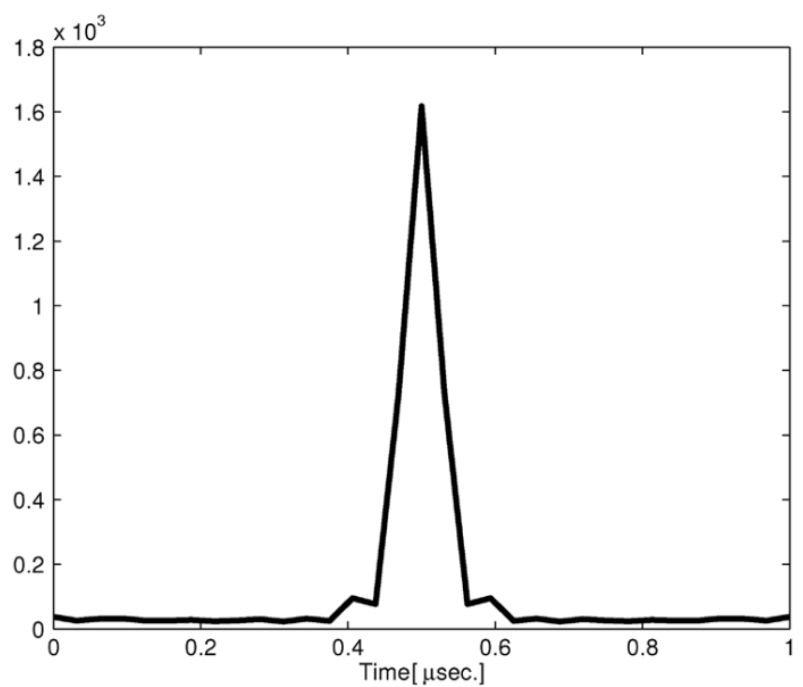

(c)

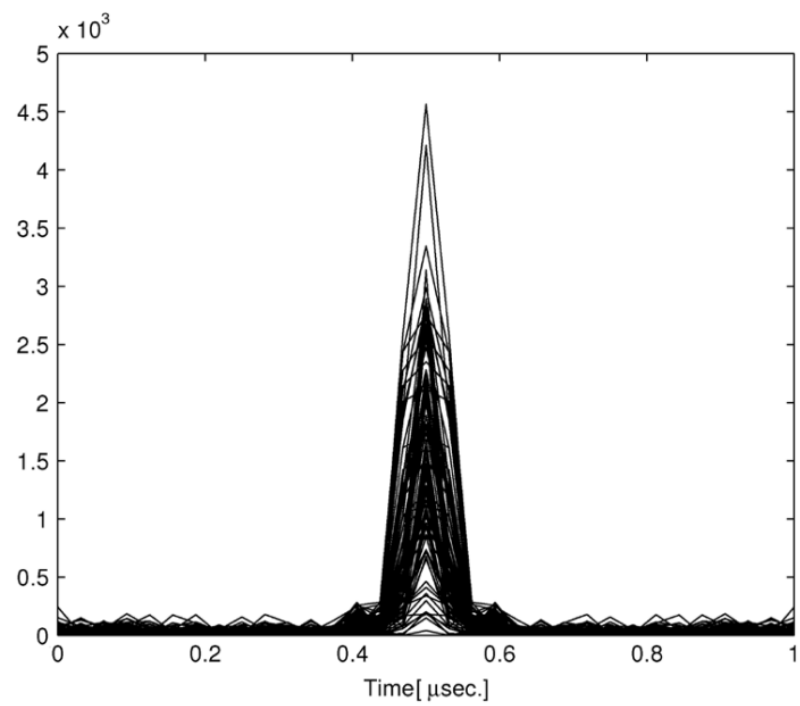

(b)

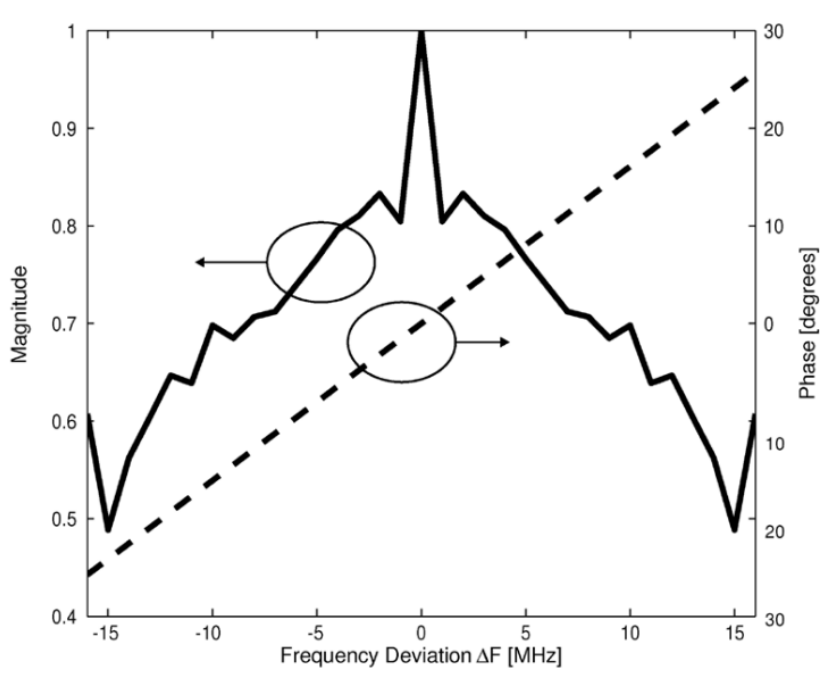

(d)

Fig. 7. Frequency Correlation Analysis, HF ( $70 \pm 8 \mathrm{MHz}$ ) band. (a) Time domain response, HF band for 100 spatial samples, before correction for PLL phase shift. (b) Time domain response, HF band for 100 spatial samples, after correction for PLL phase shift. (c) Averaged (corrected) time domain response, HF band. (d) Frequency correlation function, HF band.

function of time. Therefore, (5) represents a linear relation between time and frequency. This means that $\phi_{\mathrm{PLL}}$ is a linear function of both time and frequency. Knowing the behavior of this phase response allows us to take out its effect. In order to remove these delays, a frequency correlation is first performed on each spatial sample, and then an inverse discrete Fourier transform (IDFT) is applied to transform the data in the time domain. These phase delays are mapped into the time domain as time shifts. Recognizing that the free space time shift is constant for all spatial samples, the time domain impulses are now simply shifted to a common reference, and averaging is done over the spatial samples, in the time domain. Taking the discrete Fourier transform (DFT) of this function results in the corrected frequency correlation function.

In order to demonstrate this time shift, multipath effects are ignored and the received signal for each spatial sample, is represented by

$$
S_{r}(f)=S^{\prime}(f) e^{j\left(2 \pi f \tau_{\mathrm{fs}}+\phi_{\mathrm{ch}}(f)+\phi_{\mathrm{PLL}}(f)\right)}
$$

where $S^{\prime}(f)$ is a complex amplitude function, $\tau_{\mathrm{fs}}$ represents the free space propagation delay, $\phi_{\mathrm{ch}}(f)$ represents the frequency dependent phase characteristics of the channel (as well as phase noise caused by the PLLs, which will be discussed later in this section) and $\phi_{\mathrm{PLL}}(f)$ represents the phase shift caused by the relative phase differences between the PLLs of the VNAs. Calculating the frequency correlation function of (6), results in the following:

$$
c_{s}(\Delta f)=\int_{-\infty}^{\infty} S_{r}(f) S_{r}^{*}(f+\Delta f) d f
$$

which, upon inserting (6) in (7), becomes

$$
\begin{aligned}
c_{s}(\Delta f)= & \int_{-\infty}^{\infty} S^{\prime}(f) S^{\prime *}(f+\Delta f) e^{j\left(\phi_{\mathrm{ch}}(f)-\phi_{\mathrm{ch}}(f+\Delta f)\right)} \\
& \times e^{-j 2 \pi \Delta f\left(\tau_{\mathrm{fs}}+\tau_{\mathrm{PLL}}\right)} d f \\
= & e^{-j 2 \pi \Delta f\left(\tau_{\mathrm{fs}}+\tau_{\mathrm{PLL}}\right)} c(\Delta f)
\end{aligned}
$$




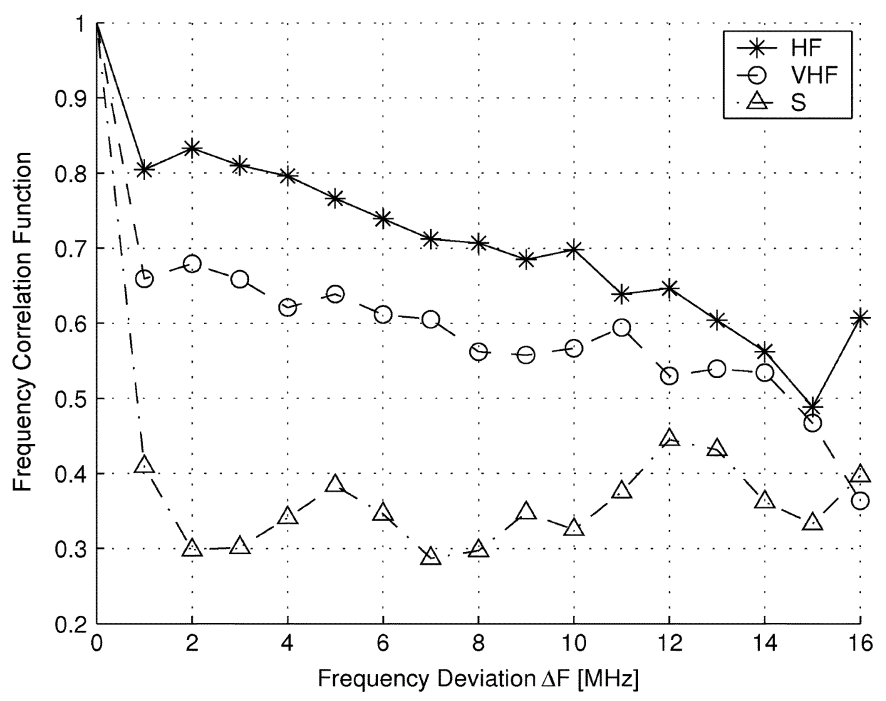

Fig. 8. Comparison of the frequency correlation functions for HF, VHF, and $S$-bands.

Taking the Fourier transform of (8) results in

$$
\begin{aligned}
\tilde{C}_{s}(T) & =\mathrm{FT}\left\{e^{-j 2 \pi \Delta f\left(\tau_{\mathrm{fs}}+\tau_{\mathrm{PLL}}\right)} c(\Delta f)\right\} \\
& =\tilde{C}\left(T-\tau_{\mathrm{fs}}-\tau_{\mathrm{PLL}}\right)
\end{aligned}
$$

where $c(\Delta f)$ and $\tilde{C}(T)$ represent a Fourier transform pair. The variable time delays (caused by $\tau_{\mathrm{PLL}}$ ) can be observed in the time domain plots in Fig. 7(a), for the upper HF-band measured $(70 \pm 8 \mathrm{MHz})$. In this figure, for measurements taken at site $\mathrm{Rx} 2$ (approximately $1.1 \mathrm{~km}$ from the transmitter, see Fig. 5), the raw frequency domain data was adjusted to remove the free space phase delay of $1.1 \mathrm{~km}$, a frequency correlation performed, and a IDFT applied to the resulting correlation function. The free space phase delay is removed to prevent aliasing in the time domain. For these measurements, the data is sampled in frequency increments of $1 \mathrm{MHz}$, thus the alias free range of the time domain data is $300 \mathrm{~m}$. By normalizing the frequency data to the free space phase delay, the alias free range is localized around the receiver. If the delay spread (in distance) between the direct and multipath signal components is less than $300 \mathrm{~m}$ (a valid assumption over the distances involved), the transformed data can be assumed to be alias free.

The shift in the observed peaks of the traces in Fig. 7(a) correspond to the phase shift caused by the unknown relative phases of the PLLs. It is now a simple matter to shift each peak to a common reference, as shown for all spatial samples in Fig. 7(b), and average the spatial samples in the time domain, as shown in Fig. 7(c). Thus the effect of random phase shift, observed in each spatial sample, is mitigated. An FFT is then performed to obtain the desired corrected frequency correlation function and the results is presented in Fig. 7(d).

In order to generate an accurate frequency correlation function, another factor must also be considered. This is the inherent system decorrelation that is caused by phase noise in the error signal (output) of the PLLs. Ideally, if one were to connect the transmit and receive sections of the measurement system together, gather a data set, and perform a frequency correlation, one would see a perfectly correlated channel. In practice, due to this phase noise in the PLLs, the system transfer function would not show perfect frequency correlation. To account for decorrelation caused by this system phase noise, it is recognized that the channel and system frequency correlation functions are statistically independent from one another, and therefore the correlation function of the measured signal is simply the multiplication of the correlation function of the system and that of the channel, or

$$
R_{r}(\Delta F)=R_{\mathrm{sys}}(\Delta F) R_{\mathrm{ch}}(\Delta F) .
$$

To correct for the system decorrelation then, a calibration data set is taken by connecting the transmit and receive sections together. Equation (10) is then applied, and the measured data set simply normalized by this calibration data set. Note that this calibration for the system decorrelation has been applied to data shown Fig. 7 as well as all results which follow.

Three sets of data, each selected from HF $(70 \pm 8 \mathrm{MHz})$, VHF $(493 \pm 23 \mathrm{MHz})$, and $S$-band $(1872 \pm 16 \mathrm{MHz})$, respectively, are used for the frequency correlation analysis. All data in this section is collected from site Rx2. Fig. 8 shows the frequency correlation functions for the three bands, overlapped for comparison. As can be observed in this plot there is a narrowing of the frequency correlation bandwidth for increased frequency. This is expected, as the dimensions of scatterers in the propagation environment (tree trunks, branches, twigs, leaves, short vegetation) become electrically larger, the random scattered field dominates, and decorrelates the received signal.

\section{CONCLUSION}

In this paper a novel, ultrawide-band measurement system, for communications channel characterization was presented. The system is based on the application of two VNAs, one as a transmitter and one as a receiver, and whose frequency sweeps are synchronized by two RbAC atomic clocks. These clocks also provide an extremely stable frequency reference for the VNAs. The use of the VNAs and their ability to perform swept frequency measurements, with a narrowband IF filter allows for long distance, high sensitivity propagation measurements. For an IF bandwidth setting on the receiver NWA of $3 \mathrm{kHz}$, (it can be set as low as $3 \mathrm{~Hz}$ ), and assuming a transmitter power of $10 \mathrm{~W}$, the maximum measurable loss of the system, from $30 \mathrm{MHz}$ to $3 \mathrm{GHz}$, was shown to exceed $115 \mathrm{~dB}$.

This coherent system allows for measuring the attenuation and phase characteristics of the medium and studying temporal and frequency decorrelations. In order to demonstrate some of these capabilities, data was presented and analyzed from a measurements campaign at the Lakehurst Naval Air Station, in Lakehurst, NJ. It was shown that the slope of the attenuation of the mean power, caused by a forested area, tended to decrease with increased frequency, as multiple scattering effects within the forest medium began to dominate the path loss. Also frequency correlation analysis of the measured data was given. To perform this analysis, it was shown that a phase shift, caused by the phase difference in the independent PLLs in the system, must be accounted for, and a method was presented to correct for this shift. After also correcting for the inherent system decorrelation, caused by phase noise in the PLL error signal, frequency correlation data for three different sub-bands was presented, for a forested area at the Lakehurst 
base. It was shown that for higher frequency bands, the signal decorrelated at a faster rate, in frequency, as expected, due to the increased effect of multipath on the received signal, as the forest components become electrically larger.

\section{ACKNOWLEDGMENT}

The authors would like to thank A. Hartz, B. Lyons, and W. Walker, for their assistance in conducting the measurements campaign at Lakehurst.

\section{REFERENCES}

[1] Y. Okumura, E. Ohmori, T. Kawano, and K. Fukuda, "Field strength and its variability in VHF and UHF land mobile service," Rev. Elec. Comm. Lab., vol. 16, p. 825, Sep.-Oct. 1968.

[2] N. C. Rogers, A. Seville, J. Richter, D. Ndzi, N. Savage, R. F. S. Caldeirinha, A. K. Shukla, M. O. Al-Nuaimi, K. Craig, E. Vilar, and J. Austin, "A generic model of 1-60 GHz radio propagation through vegetationfinal report," Document produced by QinetiQ for the U.K. Radiocommunications Agency, Tech. Rep., May 2002.

[3] A. G. Longley and P. L. Rice, "Prediction of Tropospheric radio transmission loss over irregular terrain, a computer method-1968," ESSA Research Laboratories, U.S. Government Printing Office, Washington, DC, Tech. Rep. ERL79-ITS67, 1968.

[4] K. Sarabandi and I. Koh, "An overview of physics-based wave propagation in forested environment," in Proc. Int. Workshop on Wave Propagation, Scattering, and Emission (WPSE03): Theory, Experiments, Numerical Simulation, and Inversion, Shanhai, China, Jun. 1-4, 2003. (invited).

[5] F. Wang, I. Koh, and K. Sarabandi, "Theory and measurements of millimeter-wave propagation through foliage," in Proc. USNC/URSI Symp., Columbus, OH, Jun. 22-27, 2003.

[6] F. Aryanfar, I. Koh, and K. Sarabandi, "Physics based ray-tracing propagation model for sub-urban areas," in Proc. IEEE AP-S Symp., vol. 4, Columbus, OH, Jun. 22-27, 2003, pp. 903-906.

[7] L. Pierce and K. Sarabandi, "Parallelized physics-based foliage wave propagation mode," in Proc. 2003 EMCC Annu. Meeting, Hampton, VA, May 20-22, 2003.

[8] I. Koh and K. Sarabandi, "Polarimetric channel characterization of foliage for performance assessment of GPS receivers under tree canopies," IEEE Trans. Antennas Propag., vol. 50, no. 5, pp. 713-726, May 2002.

[9] K. Sarabandi and I. Koh, "Effect of canopy-air interface roughness on HF-VHF propagation in forest," IEEE Trans. Antennas Propag., vol. 50, no. 1, pp. 111-122, Jan. 2002.

[10] - "A complete physics-based channel parameter simulation for wave propagation in a forest environment," IEEE Trans. Antennas Propag., vol. 49, no. 2, pp. 260-271, Feb. 2001.

[11] I. Koh, F. Wang, and K. Sarabandi, "Estimation of coherent field attenuation through dense foliage including multiple scattering," IEEE Trans. Geosci. Remote Sensing, vol. 41, no. 5, pp. 1132-1135, May 2003.

[12] M. D. Casciato, S. Oveisgharan, and K. Sarabandi, "Radio wave propagation in the presence of a coastline," Radio Sci.. Accepted for publication.

[13] M. D. Casciato and K. Sarabandi, "High frequency radio wave diffraction from singly curved surfaces-A heuristic approach," Proc. Inst. Elect. Eng.-Microwave, Antennas and Propag., pt. H, vol. 151, no. 1, pp. 43-53, Feb. 2004.

[14] N. C. Currie, F. B. Dyer, and E. E. Martin, "Millimeter foliage penetration measurements," in Proc. IEEE AP-S Symp., vol. 14, Amherst, MA, Oct. 1976, pp. 93-96.

[15] F. K. Schwering, E. J. Violette, and R. H. Espeland, "Millimeter-wave propagation in vegetation: Experiments and theory," IEEE Trans. Geosci. Remote Sensing, vol. 26, no. 3, pp. 355-367, May 1988.

[16] A. Y. Nashashibi, K. Sarabandi, S. Oveisgharan, M. C. Dobson, W. Walker, and E. Burke, "Millimeter-wave measurements of foliage attenuation and ground reflectivity of tree stands at nadir incidence," IEEE Trans. Antennas Propag., vol. 52, no. 5, pp. 1211-1222, May 2004.

[17] R. J. C. Bultitude, P. Melancon, H. Zaghloul, G. Morrison, and M. Prokki, "A comparison of indoor radio propagation characteristics at $910 \mathrm{MHz}$ and $1.75 \mathrm{GHz}$," IEEE J. Sel. Areas Commun., vol. 7, no. 1, pp. 20-30, Jan. 1989.
[18] J. G. O. Moss, M. P. Fitton, A. M. Street, K. M. Brown, C. C. Constantinou, and D. J. Edwards, "Spatio-temporal variability analysis of the wideband microcellular environment," Proc. 48th Vehicular Technology Conf., VTC 98, vol. 1, pp. 293-297, May 18-21, 1998.

[19] J. Liu, C. P. Wei, D. Chang, C. Chang, and C. Cheng, "Estimating the RF attenuation rate via roadside trees with extraction techniques," in Microwave Opt. Technol. Lett.. New York: Wiley, Aug. 2001, vol. 30, pp. 242-246.

[20] C. L. Rino and J. Owen, "Wideband propagation measurements in forested media,", Fort Monmouth, NJ, Tech. Rep. under contract DAAD-07-85-C-K569, Aug. 1988. SRI International (project 8899), submitted to U.S. Army Communications-Electronics Command (CECOM).

[21] S. J. Howard and K. Pahlavan, "Measurement and analysis of the indoor radio channel in the frequency domain," IEEE Trans. Instrum. Meas., vol. 39, no. 5, pp. 751-755, Oct. 1990.

[22] H. Zaghloul, G. Morrison, and M. Fattouche, "Frequency response and path loss measurements of indoor channel," Electron. Lett., vol. 27, no. 12, pp. 1021-1022, Oct. 1991.

[23] A. M. Street, J. G. O. Moss, and D. J. Edwards, "Outdoor-indoor wideband channel study for third generation communication systems," Inst. Elect. Eng. Nat. Conf. Antennas and Propagation, pp. 128-131, Mar. 31-Apr. 11999.

[24] A. M. Street, L. Lukama, and D. J. Edwards, "Use of VNAs for wideband propagation measurements," Proc. Inst. Elect. Eng.-Commun., vol. 148, no. 6, Dec. 2001.

[25] Stanford Reasearch Systems Edwards, "PRS10 Rubidium Frequency Standard," Tech. Rep. PRS10.

[26] L. L. Foldy, "The multiple scattering of waves," Phys. Rev., vol. 67, pp. $107-119,1945$.

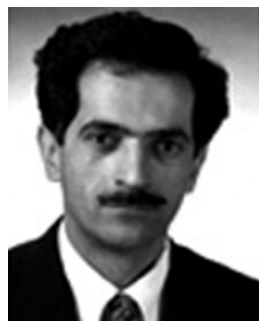

Kamal Sarabandi (S'87-M'90-SM'92-F'00) received the B.S. degree in electrical engineering from Sharif University of Technology, Tehran, Iran, in 1980, the M.S. degree in electrical engineering/mathematics, and the Ph.D. degree in electrical engineering from The University of Michigan, Ann Arbor, in 1986 and 1989, respectively.

$\mathrm{He}$ is Director of the Radiation Laboratory and a Professor in the Department of Electrical Engineering and Computer Science, The University of Michigan-Ann Arbor. He has 20 years of experience with wave propagation in random media, communication channel modeling, microwave sensors, and radar systems and is leading a large research group consisting of four research scientists, $12 \mathrm{Ph}$.D. students, and two M.S. students. Over the past ten years he has graduated $20 \mathrm{Ph}$.D. students. He has served as the Principal Investigator on many projects sponsored by NASA, JPL, ARO, ONR, ARL, NSF, DARPA, and numerous industries. He has published many book chapters and more than 115 papers in refereed journals on electromagnetic scattering, random media modeling, wave propagation, antennas, microwave measurement techniques, radar calibration, inverse scattering problems, and microwave sensors. He has also had more than 230 papers and invited presentations in many national and international conferences and symposia on similar subjects. His research areas of interest include microwave and millimeter-wave radar remote sensing, electromagnetic wave propagation, and antenna miniaturization.

Dr. Sarabandi is a Member of the International Scientific Radio Union (URSI) Commission $\mathrm{F}$ and of The Electromagnetic Academy. He received the Henry Russel Award from the Regent of The University of Michigan-Ann Arbor (the highest honor the University of Michigan bestows on a faculty member at the assistant or associate level). He received a 1996 Teaching Excellence Award from the Department of Electrical Engineering and Computer Science and a 1999 GAAC Distinguished Lecturer Award from the German Federal Ministry for Education, Science, and Technology, given to about ten individuals worldwide in all areas of engineering, science, medicine, and law. He also received the 2003/2004 College of Engineering Research Excellence Award, The University of Michigan. In the past several years, joint papers presented by his students at a number of symposia (IEEE AP'95,'97,'00,'01,'03 IEEE IGARSS'99,'02, IEEE MTTS'01) have received student prize paper awards. He is a Vice President of the IEEE Geoscience and Remote Sensing Society (GRSS), a past Chairman of the Awards Committee of the IEEE GRSS from 1998 to 2002, and a Member of the IEEE Technical Activities Board Awards Committee from 2000 to 2002. He is an Associate Editor of the IEEE TRANSACTIONS ON ANTENNAS AND PROPAGATION and the IEEE SENSORS JOURNAL. He is listed in American Men \& Women of Science, Who's Who in America, and Who's Who in Electromagnetics. 


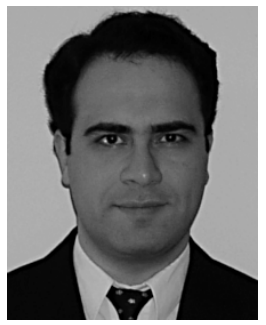

Nader Behdad (S'98) was born in Mashhad, Iran, in 1977. He received the B.Sc. degree from Sharif University of Technology, Tehran, Iran, in 2000 and the M.Sc. degree from the University of Michigan, Ann Arbor, MI, in 2003, where he is currently working toward the Ph.D. degree in the Department of Electrical Engineering and Computer Science, with a focus on bandwidth enhancement and miniaturization of printed antennas.

From 2000 to 2001, he was with the Electronics Research Center, Sharif University of Technology, as an Antenna Design Engineer working on the design of antennas for wireless local loop (WLL) systems. Since January 2002, he has been working as a Research Assistant in the Radiation Laboratory, University of Michigan.

Mr. Behdad is the recipient of the Best Student Paper Award in the Antenna Applications Symposium held in Monticello, IL, in September 2003 and was the winner of the second prize in the student paper competition of the USNC/URSI National Radio Science Meeting, Boulder, CO, in January 2004.

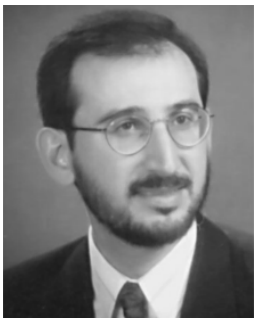

Adib Nashashibi (S'82-M'95-SM'01) received the B.Sc. and M.Sc. degrees in electrical engineering from Kuwait University, Kuwait, in 1985 and 1988, respectively, and the Ph.D. degree in electrical engineering from The University of Michigan, Ann Arbor, in 1995.

$\mathrm{He}$ is presently an Associate Research Scientist at the Radiation Laboratory at The University of Michigan. His research interests include microwave and millimeter-wave remote sensing, polarimetric radar calibration and measurement techniques, bistatic radar phenomenology, electromagnetic wave propagation, and scattering in random media.
Mark Casciato (M'01) received the B.S.E.E. and M.S.E.E. degrees from Florida Atlantic University, Boca Raton, in 1988 and 1995, respectively, and the Ph.D. degree in applied electromagnetics from The University of Michigan, Ann Arbor, in April 2001.

From 1989 to 1992, he worked as an RF/Microwave Design Engineer at Allied Signal Aerospace, Ft. Lauderdale, FL. In summer 1995, he interned at the Lincoln Laboratory/Group 34, Massachusetts Institute of Technology, Cambridge, working in the area of electromagnetic scattering prediction. From 1995 to 2001, he worked as a Graduate Student Research Assistant at The University of Michigan. From 2001 to 2003, he worked as an Assistant Research Scientist with The University of Michigan. He is currently employed by EMAG Technologies Incorporated, Ann Arbor, MI. His current research interests include electromagnetic scattering and diffraction techniques as applied to radio wave propagation and radar, propagation phenomenology, and microwave measurements and measurement systems.

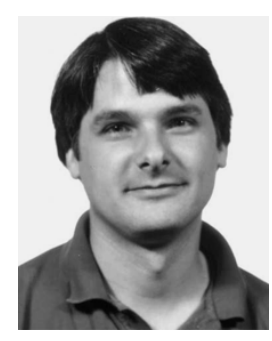

Leland Pierce (SM'00) received the B.S. degree in both electrical and aerospace engineering and the M.S. and Ph.D. degrees in electrical engineering all from the University of Michigan, Ann Arbor, in 1983, 1986, and 1991, respectively.

Currently, he is the Head of the Microwave Image Processing Facility within the Radiation Laboratory, Electrical Engineering and Computer Science Department, University of Michigan, where he is responsible for research into the uses of polarimetric SAR systems for remote sensing applications, especially forest canopy parameter inversion

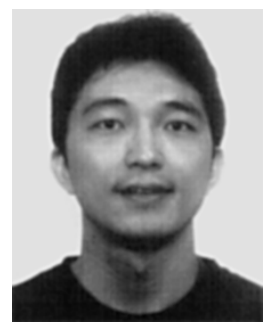

Feinian Wang (S'02) was born in China. He received the B.S. degree in physics from Tsinghua University, Beijing, China, in 1998 and the M.S. degree in electrical engineering from the University of Houston, Houston, TX, in 2000 . He is currently working toward the Ph.D. degree in electrical engineering at the Radiation Laboratory at the University of Michigan, Ann Arbor:

In 2001, he worked as an RF Engineer in Comsearch, Northern Virginia. His research interests include theoretical modeling and measurements of wave propagation, numerical techniques for random media scattering, radar and Interferometry systems. 